\title{
The Basis and Potentials of Ogunlade Sex Determination Electrocardiographic Score (OSDES) in Young Adults
}

\author{
Oluwadare Ogunlade*, Muritala Abiola Asafa \\ Department of Physiological Sciences, Obafemi Awolowo University, Ile-Ife, Nigeria
}

Email address:

oogunlade@oauife.edu.ng (O. Ogunlade),drashafa@gmail.com (M. A. Asafa)

To cite this article:

Oluwadare Ogunlade, Muritala Abiola Asafa. The Basis and Potentials of Ogunlade Sex Determination Electrocardiographic Score (OSDES) in Young Adults. Clinical Medicine Research. Vol. 4, No. 2, 2015, pp. 58-62. doi: 10.11648/j.cmr.20150402.16

\begin{abstract}
The potentials of electrocardiogram (ECG) in sex determination in young adults have not been extensively explored despite the fact that ECG is one of the most frequently performed medical procedures. This study aimed at developing a simple electrocardiographic score system that could assist in sex determination in young adults. Patterns of ECG in male and female young adults were carefully analyzed and features that could be used to delineate the ECG into male and female patterns were selected as criteria for formulation of a simple scoring system called Ogunlade sex determination electrocardiographic score (OSDES).Components of OSDES were point scores in four electrocardiographic parameters; T wave configuration in V1 (1-3), ST segment in V2 or V3 (1-3), QRS rotation (1-3) and heart rate (1-3). The total minimum and maximum scores were 4 and 12 respectively. An individual with a total score of $<7$ was identified as a female while an individual with a score $\geq 7$ was identified a male. Thus, with OSDES, ECG can be classified into two; male pattern and female pattern for male and female respectively. This unique scoring system was tested among 474 healthy young adults (294 males and 180 females). Two hundred and eighty eight (288) of the 294 males were correctly identified by OSDES as males while 174 of the 180 females were correctly identified as females $(\mathrm{k}=0.946, \mathrm{p}<0.001)$. The study defined male and female ECG patterns and concluded that ECG can be used to determine sex among young adult population.
\end{abstract}

Keywords: Basis, Potentials, Ogunlade Sex Determination Electrocardiographic Score, Young Adults

\section{Introduction}

The potentials of electrocardiogram (ECG) in sex determination in young adults have not been extensively explored despite the fact that ECG is one of the most frequently performed medical procedures and sex differences in surface ECG have been known for decades [1-2].Significant sex differences had been demonstrated with regards to heart rate, intervals, amplitude of waves of QRS complex, QRS transition zone and ST segment pattern [3-6]. Non-invasively, prenatal sex determination by ultrasonography is well known and is commonly done for women who desire to know the sex of the baby in utero [7-8]. In young adult population, sex determination had been done non-invasively using skull morphological traits such as supraorbital ridges, mastoid processes, cheek bones, orbit and mandible [9]. Invasive sex determination technique involves the use of chromosomal analysis. This technique will accurately determine the chromosomal sex but it's mostly not available in most health institutions especially in developing countries. In most cases, sex of individual is determined by the outlook or physical appearance of the person or his or her documentation with regards to his or her sex. This approach is highly subjective. Therefore, there is need to explore non-invasive objective and simple method of sex determination especially among young adult population. This is important in the light of possibility of the presence of genetic or chromosomal anomalies from the time of conception which can adversely affect sex differentiation process resulting in sex identity disorders in the young adult population. Identifying individual to be male or female by physical appearance, personal record, gender role or sexual orientation only without objective assessment may not be sufficient. This study therefore aimed at utilizing electrocardiographic characteristics distinguishing males from females to develop a simple scoring system for sex determination in young adults. 


\section{Methods}

\subsection{Participant Selection}

The target population was students of the Obafemi Awolowo University, Ile-Ife between the ages of 17 and 44years. A total of 500 subjects participated in the study after the initial clinical screening process. It was a cross-sectional descriptive study. The ethical clearance was obtained from the Ethics and Research Committee of the Obafemi Awolowo University Teaching Hospitals Complex Ile-Ife. Volunteers for the study were evaluated clinically to exclude symptomatic systemic diseases that could adversely affect the cardiovascular status. The clinical screening entailed history taking and physical examination.

\subsection{ECG Acquisition Procedure}

The standard 12-lead resting ECG of each participant was obtained in the supine position during quiet respiration. The participants were educated about the procedure and written informed consent obtained from them. Materials like wrist watch, jewellery, coins and mobile phones were removed from the body to reduce electromagnetic interference and improve the quality of the ECG. The chest and the limbs were exposed and ECG electrode placed according to internationally approved protocol [10]. The chest lead recordings (V1-V6) were obtained by the attachment of 6 electrodes to the anterior chest wall according to the conventional method; V1 at 4th intercostal space right sternal edge, $\mathrm{V} 2$ at 4 th intercostal space left sternal edge, $\mathrm{V} 3$ at the point mid-way between $\mathrm{V} 2$ and $\mathrm{V} 4$, $\mathrm{V} 4$ at 5th intercostal space left midclavicular line, V5 at 5th intercostal space left anterior axillary line and V6 at 5th intercostal space left mid-axillary line. The six limb leads (I, II, III, aVF, aVL and aVR) were recorded by the aids of four electrodes attached to the distal end of each limb according to the standard protocol for limb electrode placement [10]. The ECG technician entered the identification number, age, sex, weight, height and blood pressure into the ECG machine. The standard 12-lead ECGs were recorded by ECG technician at a speed of $25 \mathrm{~mm} / \mathrm{s}$ and calibration signal of $10 \mathrm{~mm} / \mathrm{mV}$. The results were printed out.

\subsection{Development of the Ogunlade Sex Determination Electrocardiographic Score}

Out of the 500 ECGs, a total of 26(13 males and 13 females) were selected and analyzed to document the specific electrocardiographic features distinguishing male from female ECGs based on the expertise and experience of the cardiologist. These sex specific features were given scores which were sum-up. These features were used to develop a simple scoring system called Ogunlade Sex Determination Electrocardiograhic Score (OSDES). Components of OSDES were; T wave configuration in V1 (1-3), ST segment in V2 or V3 (1-3), QRS rotation (1-3) and heart rate (1-3). The total minimum and maximum scores were 4 and 12 respectively. Appropriate cut-off point of 7 was set. A female ECG pattern was distinguished by a total score $<7$ while male ECG pattern was distinguished by a total score $\geq 7$ (Table 1$)$.

\subsection{Utilization of Ogunlade Sex Determination Electrocardiographic Score}

The remaining four hundred and seventy four (474) ECGs (not considered at the developmental stage of the OSDES) were serialized by the ECG technician with information about sex properly recorded. Subsequently, the ECG technician utilized a black cellotape to cover the sex segment of each of the ECGs. A cardiologist and a physician assistant who were blinded to the previous sex record of the ECGSs objectively utilized OSDES to determine the sex of the 474 ECGs one after the other.

\subsection{Statistical Analysis}

The data were analyzed using SPSS version 17. Descriptive analysis was performed on the patients' biodata. The sex determined by OSDES was compared with the sex recorded by the ECG technician using kappa statistics.

\section{Results}

A total of 474 young adults ECGs were scored using OSDES The mean $( \pm \mathrm{SD})$ height, weight and body mass index of the participants were $1.68 \pm 0.83 \mathrm{~m}, 66.3 \pm 9.05 \mathrm{~kg}$ and 21.82 $\pm \quad 2.80$ respectively. Dominant electrocardiographic characterristics of male ECG were; upright $\mathrm{T}$ wave in lead V1, ST segment elevation ( $\geq 2 \mathrm{~mm}$ above PR segment) in leads V2-V3, normal transition (transition zone) at V3-V4 and a lower heart rate usually $<70$ beats per minute[Figure 1]. Dominant electrocardiographic characteristics of female ECG were; $\mathrm{T}$ wave inversion or flattening lead V1, isoelectric ST segment(within $1 \mathrm{~mm}$ of PR segment) in V2-V3, anticlockwise or counter clockwise rotation and a higher heart rate usually $>70$ beats per minute [Figure 2]. Among the 474 participants, 288 of the 294 males were correctly identified by OSDES as males while 174 of the 180 females were correctly identified as females (Table 2). The agreement between the sex determined by OSDES and sex recorded by ECG technician was almost perfect $(\mathrm{k}=0.946, \mathrm{p}<0.001)$. 
Table 1. Ogunlade Sex Determination Electrocardiographic Score (OSDES).

\begin{tabular}{|c|c|c|c|}
\hline \multicolumn{2}{|c|}{ Parameters } & \multirow{2}{*}{$\begin{array}{l}\text { Criteria } \\
\text { Inversion }\end{array}$} & \multirow{2}{*}{$\frac{\text { Score }}{1}$} \\
\hline & & & \\
\hline \multirow[t]{3}{*}{1} & $\mathrm{~T}$ wave in $\mathrm{V} 1$ & Flattened & 2 \\
\hline & & Upright & 3 \\
\hline & & Isoelectric: at $0-1 \mathrm{~mm}$ of $\mathrm{PR}$ segment & 1 \\
\hline \multirow[t]{3}{*}{2} & ST segment in V2 or V3 & Depression $: \geq 2 \mathrm{~mm}$ below PR segment & 2 \\
\hline & & Elevation : $\geq 2 \mathrm{~mm}$ above PR segment & 3 \\
\hline & & Anticlockwise rotation & 1 \\
\hline \multirow{2}{*}{3} & & Clockwise rotation & 3 \\
\hline & & $>70$ & 1 \\
\hline \multirow[t]{2}{*}{4} & Heart rate (beats per minute) & $60-70$ & 2 \\
\hline & & $<60$ & 3 \\
\hline \multicolumn{2}{|c|}{ Total } & & $4-12$ \\
\hline
\end{tabular}

OSDES $<7=$ Female, OSDES $\geq 7=$ Male

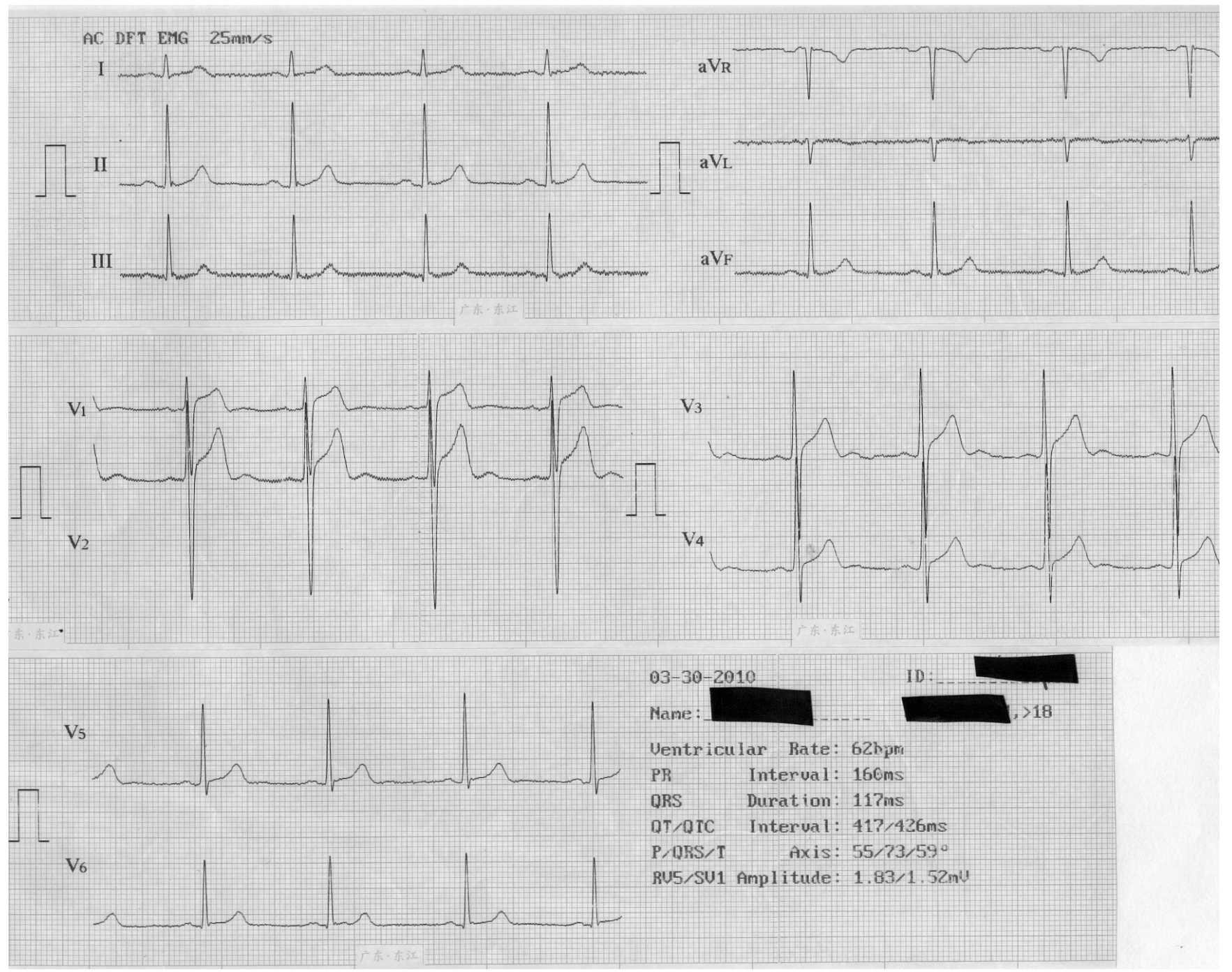

Figure 1. ECG of 27year old Nigerian showing electrocardiographic features of male ECG pattern; Upright T wave in V1(3),elevation of ST segment in V2 or $V 3(3)$, normal transition (2) and heart rate between 60 and 70 beats per minute(2). The total OSDES $=10$. 

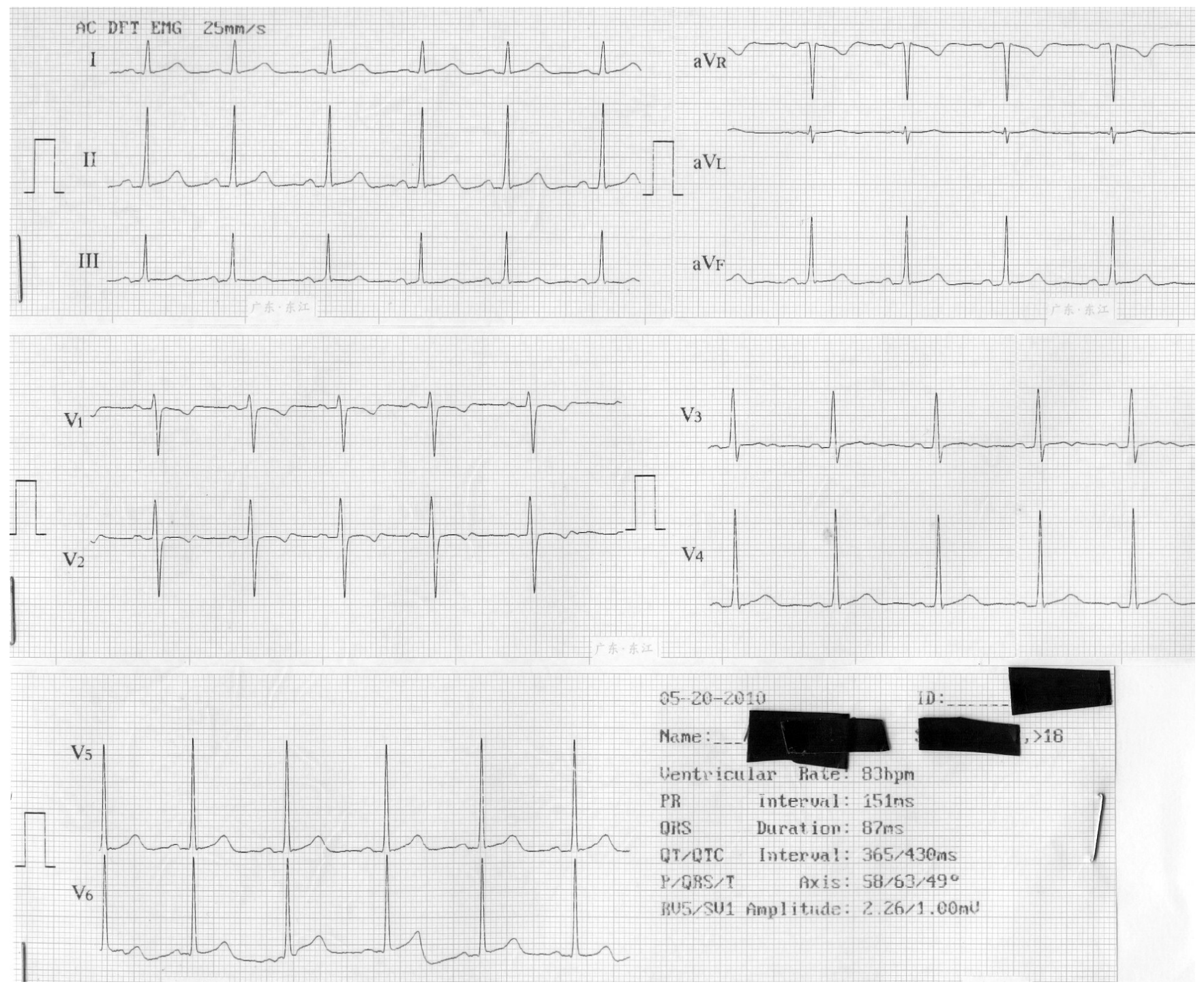

Figure 2. ECG of 24year old Nigerian showing electrocardiographic features of female ECG pattern; Inverted T wave in V1(1), isoelectric ST segment in V2 or $V 3(1)$, anticlockwise rotation(1) and heart rate $>70$ beats per minute(1). The total OSDES $=4$.

Table 2. Comparison between OSDES identified sex and sex recorded by ECG technician.

\begin{tabular}{llll}
\hline \multirow{2}{*}{ OSDES Sex } & \multicolumn{2}{l}{ Sex Recorded by ECG Technician } & \multirow{2}{*}{ Total } \\
\cline { 2 - 3 } & Male & Female & \\
\hline Male & 288 & 6 & 294 \\
Female & 6 & 174 & 180 \\
Total & 294 & 180 & 474 \\
\hline
\end{tabular}

$\mathrm{k}=0.96, \mathrm{p}<0.001$. There was almost a perfect agreement between the two methods of sex identification.

\section{Discussion}

The first recorded human ECG was published by a British Physiologist, Augustus D Waller of St Mary's Medical School, London [11]. However, Willem Einthoven, a Dutch physiologist opened up the field of clinical application of ECG. He published the first organized presentation of normal and abnormal ECG[12].Einthoven described left and right ventricular hypertrophy, left and right atrial hypertrophy, the $\mathrm{U}$ wave, notching of the QRS complex, ventricular premature beats, ventricular bigeminy, atrial flutter and complete heart block [12].

In modern clinical practice, ECG has a wider application in the evaluation of various forms of cardiovascular disorders such as left ventricular hypertrophy, right ventricular hypertrophy, right and left atrial abnormalities, heart failure, hypertension, coronary artery diseases, rheumatic valvular diseases, cardiomyopathies, congenital heart diseases, acute and chronic cor pulmonale, myocarditis, pericarditis and pericardial effusion [13]. ECG is the gold standard non-invasive test for the clinical diagnosis of cardiac arrhythmias [14] and heart block [15-16). The use of ECG in gender determination in the young has not been previously demonstrated until inception of this study. However, gender differences in ECG of young adults had been established [4]. In particular of note was early repolarization which had been demonstrated as dominant feature of male ECG especially among young black population [4]. Surawicz et al,2002 demonstrated male and female pattern of ECG based on pattern of $\mathrm{J}$ point and ST angle. The female pattern was 
characterized by $\mathrm{J}$ point $<0.1 \mathrm{mV}$ in each of the leads $\mathrm{V} 1-\mathrm{V} 4$ while the male ECG pattern was characterized by $\mathrm{J}$ point $\geq$ $0.1 \mathrm{mV}$ and the ST angle $\geq 20^{\circ}$. An indeterminate pattern was described if the $\mathrm{J}$ point was $\geq 0.1 \mathrm{mV}$ and the $\mathrm{ST}$ angle $<$ $20^{0}[17]$. In a study of 660 healthy young adults between the ages of 18-29 years, Scherptong et al, 2008 demonstrated significant sex differences which reflected in the spatial QRS-T angle[18].Moreover, Hingorani et al, 2012 also demonstrated significant sex differences in morphological abnormalities in baseline ECG of healthy adults in India. The study showed that sinus bradycardia, first degree atrioventricular block, right bundle branch block, intraventricular conduction defects and left axis deviation were more common in males while sinus tachycardia, short PR interval and non-specific ST segment and T wave changes were more frequent in females[19]. A gender-based analysis of high school athletes using computerized ECG measurement by Kumar et al, 2013 demonstrated significant sex differences which included a longer QRS duration, PR interval, ST interval and $Q$ wave in male athletes and a longer QTc interval in female athletes. All these study confirmed that ECG could be used to distinguish male from female young adult. It is noteworthy that the basis for sex differences in cardiac development and electrophysiology appears to be genetic coupled with strong hormonal and autonomic influence [21].

In conclusion, the present study demonstrated that ECG can be used to determine sex among healthy young adult population.

\section{Acknowledgements}

We are grateful for the technical support of the members of staff of Department of Physiological Sciences, Obafemi Awolowo University, Ile-Ife.

\section{References}

[1] H. Bazzet. An analysis of the time relations of electrocardiograms.Heart.1920,7 pp353-370.

[2] R.Ashman. The normal duration of the QT interval.Am Heart J 1942, 23 pp 522-534.

[3] R. M. Gowda, S. L. Wilbur, P. Schweitzer. Gender differences in cardiac electrophology and arrhythmia. Part 1, Cardiol 2006, 15(6) pp 296-302.

[4] O.Ogunlade, A.O. Ayoka, A.O. Akintomide,O.E, Ajayi, O.E. Ukponmwan, O.B. Ogunlade. Gender differences in electrocardiogram of young adults in South-Western Nigeria. Eur J Sci Res 81pp 26-32.

[5] P.M. Okin, M.J. Roman, R.B. Devereux, P. Kligfied. Gender differences and the electrocardiogram in left ventricular hypertrophy. Hypertension 1995, 25 pp 242-249.

[6] E. Natsuyama. Sonographic determination of fetal sex from twelve weeks of gestation. Am J Obstet Gynecol. 1984,149 pp748-57
[7] B. O. Igbinedion, T. O. Akhigbe. The accuracy of 2D ultrasound prenatat sex determination. Niger Med J 2012, 53(2) pp71-75.

[8] C. H. Ingerslev, B Solow. Sex differences craniofacial morphology. Acta Odontol Scand 1975, 33 (2) pp 85-94.

[9] J. R. Koch, G.G. Koch. The measurement of observer agreement for categorical data. Biometrics 1977,33 pp159-174.

[10] P. Kligfield, L. S Gettes, J.J. Bailey, R..Childers, B.J. Deal, W. Hancock et al. Recommendations for the Standardization and Interpretation of the Electrocardiogram. Part I: The Electrocardiogram and Its Technology. A Scientific Statement From the American Heart Association Electrocardiography and Arrhythmias Committee, Council on Clinical Cardiology; the American College of Cardiology Foundation; and the Heart Rhythm Society. Circulation 2007,115 pp 1306-1324.

[11] A.D. Waller. A demonstration on man of electromotive changes accompanying the heart's beat. J Physiol (London) 1887, 8 pp 229-234.

[12] W. Einthoven. Le telecardiogramme. Arch Int de Physiol 1906, 14 pp 132-164.

[13] D. M. Mirvis, A. L Goldberger. Electrocardiography. In: P. Libby, R.O. Bonow, D.L. Mann, D.P Zipes, E Braunwald.(eds). Braunwald's heart disease: a textbook of cardiovascular medicine.8th Ed. Saunders Elsevier 2008 pp149-193.

[14] C. Fisch. Evolution of the electrocardiogram. J Am Coll Cardiol 1989, 14 pp1127-1138.

[15] H. H. Hecht, C.E Kossman. Atrioventricular and intraventricular conduction. Am J Cardiol 1973, 31pp 232-244.

[16] M.E. Silverman, C.B. Upshaw, H.W. Lange. Woldermar Mobitz and His 1924 classification of second degree atrioventricular block. Circulation. 2004,110 pp 1162 -1167.

[17] B.Surawicz, SR Parikh. Prevanlence of male and female pattern of early ventricular repolarization in the normal ECG of males and females from childhood to old age. J Am Coll Cardiol 2002, 40(10)pp1870-1876.

[18] R.W. Scherptong, I.R. Henkens, S.C. Man, S.L. Cessie, H.W. Vliegen, A.C. Maan, M.J. Schalij, C.A. Swenne. Interpretation of spatial QRS-T angle: Normal limits in healthy subjects and implications for risk stratification. Circulation 2008,118 ppS_986.

[19] P. Hingorani, M. Natekar, S. Deshmukh, D .R. Karnad, S. Kothari, D. Narula, Y. Lokhandwala. Morphological abnormalities in baseline ECGs in healthy normal volunteers participating in phase I studies. Indian J Med Res 2012,135 pp 322-330.

[20] N. Kumar, D. Saini, V. Froelicher. A gender-based analysis of high school athletes using computerized electrocardiogram measurements. PLos ONE 2013,8(1) pp e53365.

[21] T.V. Pharm. Gender differences in cardiac development: are hormones at the heart of the matter?. Cardiovasc Res 2003,57 pp 591-593.

[22] X. Du. Gender modulates cardiac phenotype development in genetically modified mice. Cardiovasc Res 2004,63 pp 510-519. 